\title{
FACTORES DE RIESGO ASOCIADOS A GANGRENA DE FOURNIER
}

\author{
RISK FACTORS ASSOCIATED TO FOURNIER GANGRENA
}

Jose C. Regalado-Altamirano

\begin{abstract}
RESUMEN
Introducción: La gangrena de Fournier es una fascitis necrotizante que afecta la región perineal, perianal, genital y del tercio inferior del abdomen que lleva a necrosis y gangrena del tejido subyacente y compromete de manera fulminante y progresiva la vida del paciente. Se considera que hay diversos factores que favorecen su aparición. El objetivo de este artículo es realizar una revisión profunda sobre los factores de riesgo y las afecciones predisponentes de la gangrena de Fournier durante los últimos años. Métodos: Se realizó una revisión sistemática de artículos científicos, revisiones clínicas e informes de casos; tanto en español como en inglés, relacionados con el tema. Las fuentes de búsqueda empleadas fueron: Google Scholar, ScienceDirect - Elsevier library, PubMed, Scielo. Para esta revisión se seleccionaron los artículos publicados que tuvieron experiencias investigativas y teórico-conceptuales desde el 2008 a la actualidad. Resultados: Basado sólo en 21 de los artículos revisados, se evidenció que la gran mayoría de pacientes eran varones por ser un grupo de riesgo, y tenían una importante afección orgánica de base; destacando la diabetes mellitus, alcoholismo crónico, inmunosupresión y otras comorbilidades. Conclusión: La gangrena de Fournier es una entidad que puede ser letal, que está favorecida por distintos factores de riesgo, cuya causa desencadenante es una enfermedad debilitante así como también afecciones perirrectales o urogenitales que no han sido tratadas correctamente. Debido a su pronóstico grave, un diagnóstico temprano con un buen manejo de los factores de riesgo y una intervención multidisciplinaria adecuada, temprana y agresiva son esenciales para una buena evolución.
\end{abstract}

Palabras clave: Gangrena de Fournier; Factores de riesgo. (fuente: DeCS BIREME)

\begin{abstract}
Introduction: Fournier gangrene is a necrotizing fasciitis that affects the perineal, perianal, genital and lower third of the abdomen that leads to a necrosis and gangrene of the underlying tissue and compromises the life of the patient in a fulminant and progressive manner. It is considered that there are many factors that favor its appearance. The objective of this article is to carry out an in-depth review of the risk factors and the predisposing conditions of Fournier's gangrene during the last years. Methods: A systematic review of scientific articles, clinical reviews and case reports was carried out; both in Spanish and in English, related to the topic. The search sources were: Google Scholar, ScienceDirect - Elsevier Library, PubMed, Scielo. For this review, the published articles that had investigative and theoretical-conceptual experiences from 2008 to the present were selected. Results: Based on only 21 of the reviewed articles, it was evidenced that the great majority of patients were male because they were a risk group, and they had an important organic base condition; highlighting diabetes mellitus, chronic alcoholism, immunosuppression and other comorbidities. Conclusion: Fournier gangrene is an entity that can be lethal, which is favored by various risk factors, whose triggering cause is a debilitating disease as well as perirectal or urogenital affections that have not been treated correctly. Due to its severe prognosis, an early diagnosis with a good management of the risk factors and an adequate multidisciplinary intervention, early and aggressive, essential for a good evolution.
\end{abstract}

Key words: Fournier gangrene; Risk factors. (source: MeSH NLM)

'Facultad de Medicina Humana, Universidad Ricardo Palma, Lima - Perú.

Citar como: Jose C. Regalado-Altamirano. Factores de riesgo asociados a gangrena de Fournier. [Artículo de Revisión].2018;18(4):92-100. (Octubre 2018). DOI 10.25176/RFMH.v18.n4.1736 


\section{INTRODUCCIÓN}

La gangrena de Fournier ( $F G$ ) es una infección necrosante de rápida progresión que resulta de la acción sinérgica de múltiples aeróbicos y organismos anaeróbicos colonizados en la piel y en el área anogenital. Esto es un una condición altamente fulminante, potencialmente letal, descrita por primera vez en 1883 por un dermatólogo parisino, JeanAlfred Fournier (1832-1914), como fascitis necrosante específica del sitio que implica la áreas perineales, urogenitales y anorrectales del cuerpo ${ }^{1-4}$. Mientras que la fascitis necrotizante generalmente involucra las extremidades, FG es una variante que comienza en la región perianal o perineal y rápidamente migra a la pared abdominal o proximal muslos como resultado de la invasión en el perineal fascia. Fournier inicialmente observó esta infección en jóvenes por lo demás sanos, pero desde entonces se ha descrito en ambos sexos y en pacientes de todas las edades, con la mayor incidencia en pacientes con enfermedades cardíacas crónicas y pacientes diabéticos mal controlados ${ }^{5,6}$.

La incidencia de FG es mayor en hombres (Proporción 9-10:1), aunque las mujeres pueden estar más afectadas generalmente. En una revisión retrospectiva de pacientes tratados en una instalación terciaria desde 1996 a 2007, Barreda JT et al. ${ }^{3}$ informaron una relación similar. También, contrariamente a las suposiciones previas, el fuente de infección es más a menudo colorrectal y dermatológico que urológico. En mujeres, fuentes potenciales de FG han sido identificadas después de abortos sépticos, episiotomías y genitales con piercings. La edad media de presentación ha reportado como $54.7 \pm 15.6$ años, pero FG tiene sido informado en pacientes a lo largo de la vida lapso, aunque la incidencia aumenta con la edad ${ }^{5,7}$.

Si bien influyen una serie de factores predisponentes el pronóstico de $\mathrm{FG}$, las tasas de mortalidad se mantienen alto a pesar de una gestión agresiva. El curso clínico de FG se divide en 2 fases: la primera fase después de una precipitación incidente es relativamente estable y variable en el tiempo. En esta etapa temprana, puede haber inflamación localizada que rodea una infección cutánea o absceso, y el sistema inmune se moviliza para prevenir la propagación.

La segunda fase, sin embargo, se caracteriza por la escalada rápida del dolor y la invasión a tejido subcutáneo y planos fasciales y como progresa a una enfermedad fulminante. La infección puede extenderse a lo largo de la fascia tan rápido como 1 pulgada por hora sin evidencia externa de cambios en la piel ${ }^{8,9}$.
La mediana del tiempo desde el inicio de los síntomas hasta la presentación clínica a un hospital es de 6 días (rango, 2-28 días), con mayor mortalidad asociado con el retraso en el tratamiento de más de 48 horas.

\section{ANATOMÍA Y FISIOPATOLOGÍA}

El conocimiento de la anatomía del piso pélvico y las estructuras fasciales son importantes para la comprensión la rápida progresión de FG en todas las estructuras urogenitales y anales. La fascia de Colles es una capa membranosa de subcutánea tejido del perineo que se adhiere posteriormente a los lados de los ramos isquiópúbricos y continúa en la pared abdominal anterior como se adhiere a la fascia de Scarpa. También continua con la fascia de Colles es la fascia dartos, que contiene fibras musculares y se extiende el escroto para formar el tabique escrotal. El área periuretral y las estructuras más profundas del pene están cubiertos por la fascia de Buck. La infección puede migrar desde cada una de estas áreas primarias como el plano fascial ${ }^{9}$.

El consenso general entre los expertos es que la FG es causado por polimicrobios que actúan en sinergia para producir toxinas enzimáticas que causan una multiplicación rápida y diseminación en fascia subyacente y tejido subcutáneo.

Estos relativamente benignos, organismos naturales en la piel perineal y los órganos genitales incluyen Clostridium, Klebsiella, estreptococos, coliformes, estafilococos, Bacteroides, y corinebacterias. La infección y subsiguiente la invasión a la fascia es a menudo rápida y fulminante, pero el proceso comienza de manera similar a todos infecciones y el sistema inmune juega una clave papel en la prevención de la propagación de la necrosis. La respuesta inicial del cuerpo es la liberación de mediadores inflamatorios que causan edema local, eritema y disminución de oxígeno en los tejidos subcutáneos. La inflamación persistente resulta en necrosis tisular localizada y trombosis de pequeños vasos en la fascia. Esta falta de oxígeno en el tejido crea el ambiente óptimo para el crecimiento de los polimicrobios y liberación sinérgica de enzimas tóxicas que aceleran aún más la necrosis de tejido subcutáneo a lo largo de planos fasciales. En última instancia, hay una invasión más profunda en el músculo que causa miomiócrosis ${ }^{9}$.

\section{ETIOLOGÍA DE FG Y FACTORES PREDISPONENTES}

Históricamente, la FG se consideró como una repentina gangrena de rápida progresión, caso contrario hay algunos pacientes sanos que presentan espontáneamente esta patología en la región 
urogenital. La presentación clásica no estuvo asociado con un evento precipitante y se pensó que los casos surgieron de novo ${ }^{9,10}$.

Sin embargo, desde que fue descrito por primera vez por Fournier, la comprensión de la enfermedad ha evolucionado. En un estudio, aproximadamente el $82 \%$ de los pacientes tenía un sitio de entrada definitivo y esta tasa es coherente con los casos informados en general $^{11,12}$.

Fuentes de infección anorrectal (perianal y abscesos perirrectales) y las infecciones locales de la piel son más comunes que las entradas por puertos urogenitales (escrotal abscesos, circuncisión). La lesión inicial de la piel puede ser relativamente inocua, como una simple abrasión de la piel, contacto con agua de mar, o agua de lluvia turbia. Vibrio y Aeromonas son comunes en el agua organismos causales a menudo asociados con lesiones menores en el agua. En análisis retrospectivos de presentaciones clínicas de pacientes se indica que la presencia de comorbilidades es fuertemente asociada con ambas tasas de incidencia y pronóstico. La diabetes ha demostrado ser la condición predisponente más común, con una tasa de incidencia del $46 \%$ al $80 \%$ y mayor letalidad. En una serie de casos, todos los pacientes que murieron eran diabéticos. La etiología de esto parece ser que la infección se propaga más rápidamente en pacientes diabéticos y se presentan con signos de sepsis anteriores que los pacientes no diabéticos ${ }^{9,11,13,14}$.

Además de diabetes, otras comorbilidades asociadas con FG incluye condiciones cardíacas, sobrepeso (IMC $\geq 25$ ), obesidad (IMC $\geq 30$ ), crónica alcoholismo, inmunosupresión (crónica uso de esteroides, infección por VIH), inmovilización, enfermedad vascular periférica, desnutrición, leucemia y pulmonar obstructiva crónica enfermedad.

Los informes de estudios de casos también sugieren ese uso prolongado de inhibidores de la bomba de protones puede disminuir crónicamente la secreción de ácido gástrico y como el pH gástrico se eleva por encima de 4, hay una mayor susceptibilidad a los entéricos infecciones que incluyen Clostridium difficile, Vibrio vulnificus, y Clostridium septicum, la causa más común de gas espontáneo gangrena. Neoplasias malignas gastrointestinales ocultas debe sospecharse en pacientes donde Clostridium septicum es el organismo causal ${ }^{9,15,16}$.

\section{PRESENTACIÓNY MANIFESTACIONES CLÍNICAS DE FG}

El sello distintivo de FG es dolor intenso y sensibilidad en los genitales, aunque la clínica la presentación puede ser variable y la piel en realidad puede parecer normal en la preclínica, periodo latente. Los pacientes pueden presentarse en cualquier punto del proceso, $y$ el curso clínico parece variar considerablemente según la edad del paciente, condiciones de comorbilidad e inmunidad y estado nutricionale. Los pacientes rara vez se presentan durante la fase prodrómica, ya que los síntomas pueden ser generalizados y se manifiesta como fiebre o letargo durante días o, posiblemente, semanas. Puede haber dolor localizado o prurito, edema y menor inflamación. El paciente puede haber estado bajo tratamiento tópico en casa sin receta con remedios y analgésicos. En esta fase temprana, la infección puede ser insidiosa mientras silenciosamente va invadiendo planos fasciales. En este punto, el dolor se vuelve más intenso y desproporcionado con hallazgos físicos. A medida que se desarrolla la gangrena, el tejido nervioso se vuelve necrótico y el paciente en realidad puede experimentar menos dolor. Como necrosis progresa más, hay más sistémico efectos y el desarrollo de shock séptico.

Dentro de las características clínicas adicionales sugestivas de FG debemos incluir taquicardia, purulento, foul (feculento) oler la secreción perineal, crepitación (54.3\%), y fiebre (41.4\%). Crepitación del tejido inflamado es común e indicativo de formación de gas por organismos como Clostridium septicum.

La formación de ampollas y ampollas son hallazgos tardíos en FG y la ausencia de linfadenitis es característico de FG y fascitis necrosante. Los pacientes en las últimas etapas pueden mostrar signos de shock séptico o síndrome de shock tóxico y falla multiorgánica (hipotermia, hipotensión, confusión, arritmias cardíacas) ${ }^{17,18}$. En el examen físico de la piel en pacientes con FG se puede clasificar en 3 etapas: etapa de eritema con sensibilidad de la piel que se extiende más allá del área de hinchazón y calor; etapa II, formación de ampollas/ampollas y fluctuante piel; etapa III, crepitación, necrosis de la piel y bullas hemorrágicas ${ }^{9}$.A pesar de ser extensas afectación del escroto y el perineo los testículos pueden ser preservados si el origen de la infección es perianal o perirrectal, y testicular la participación es indicativa de un retroperitoneal o fuente de infección intraabdominal. La invasión de tejido involucra el pene, el escroto y abdomen anterior, la necrosis puede extenderse al tórax, clavículas y axilas.

La gangrena de Fournier representa una verdadera cirugía emergencia, y el tiempo es esencial una vez que aparecen los signos clínicos.

Diagnóstico diferencial: Distinguir FG de la celulitis necrosante. 
El diagnóstico precoz de FG puede ser clínicamente difícil e incluso un médico astuto puede tener dificultad para distinguir la fascitis necrosante de la celulitis necrosante, ya que ambos pueden presentar con eritema, calor, dolor $y$, posiblemente, fiebre. Característica de FG y necrosante fascitis, el dolor es desproporcionado al físico hallazgos, mientras que la celulitis necrosante puede aparecen aún más "enojados". Hay algunos distinguir los hallazgos físicos que apuntan hacia el diagnóstico de FG y fascitis necrosante, que se originan en la grasa subcutánea y fascia profunda, a diferencia de necrosante celulitis, que se desarrolla en la unión entre la dermis y la fascia superficial ${ }^{19}$.

\section{Clasificación (profundidad del tejido)}

Las infecciones necrotizantes de partes blandas se clasifican en una variedad de formas. Un método es clasificarlos por la profundidad de la infección de infecciones superficiales de la epidermis y dermis a infecciones profundas de la fascia y músculo. La profundidad de los rangos de infección tisular de infecciones superficiales de la epidermis y la dermis, como la celulitis necrosante a infecciones profundas que involucran la fascia profunda tales como FG.

\section{Tipos (fuente microbiana de la infección)}

Los tipos de infección necrotizante se basan en la causa microbiana de la infección. Polimicrobiano las infecciones (tipo I) son las más comunes tipo de infecciones necrotizantes que representan $70 \%$ a $80 \%$ de los casos, mientras que las infecciones que son mono-microbianos (tipo II), que representan $20 \%$ a $30 \%$ de los casos, son mucho menos comunes que las infecciones de tipo $\mathrm{I}^{1,9,20}$.

Las infecciones de tipo I ocurren con frecuencia en el áreas del troncoyperinealesdeinmunocomprometidos pacientes. Las infecciones tipo l, que son polimicrobianos en etiología, a menudo tienen promedio de 4 organismos diferentes. Infeccioso los microbios a menudo incluyen combinaciones de cocos grampositivos, bacilos gramnegativos y anaerobios Los microbios incluyen grampositivos organismos tales como Staphylococcus aureus, Streptococcus pyogenes y enterococos; organismos gramnegativos incluyen Escherichia coli y Pseudomonas; mientras que anaeróbico los organismos incluyen Bacteroides o Clostridium. Pacientes inmunocomprometidos a menudo incluyen factores de riesgo tales como diabetes mellitus, enfermedad vascular periférica, abuso de alcohol, obesidad, insuficiencia renal crónica, trauma contundente o penetrante, picaduras de insectos, incisiones quirúrgicas y catéteres permanentes.
Las infecciones tipo II, menos comunes que las infeccionesdetipolsoncausadaspormonomicrobianos infecciones tales como Streptococcus del grupo A (Streptococcus pyogenes) con o sin Staphylococcus aureus a menudo en las extremidades del cuerpo en individuos inmunocompetentes que tienen un historial de trauma menor reciente o la operación del tejido involucrado. Tipo II son las únicas infecciones de tejidos blandos necrosantes que están asociados con el síndrome de shock tóxico ${ }^{9}$.

Las infecciones de tipo III son poco comunes, pero a menudo resultado en la falla de órganos multisistema y un alto tasa de mortalidad incluso con diagnóstico precoz y tratamiento agresivo si no se reconoce dentro de 24 horas. Los microbios tipo III son gramnegativos infecciones, siendo la bacteria Vibrio la causa más común. Las infecciones tipo IV son muy raro y causado por infecciones fúngicas de Candida, que ocurre más comúnmente en quemar pacientes y aquellos que están gravemente inmunocomprometido.

\section{ESTUDIOS DIAGNÓSTICOS}

El diagnóstico de fascitis necrosante requiere una alto índice de sospecha desde las primeras etapas de celulitis necrosantes. La clave en el diagnóstico de fascitis necrosante en sus primeras etapas es la cantidad desproporcionada de dolor severo en comparación con los hallazgos físicos menores.

El estándar de oro en el diagnóstico de la necrosis fascitis es una biopsia de tejido quirúrgico. La media del momento del diagnóstico puede variar de 2 días a varias semanas ${ }^{9}$.

\section{TRATAMIENTO}

La sepsis es la principal causa de muerte en la unidad de cuidados intensivos (UCI). Un diagnóstico exitoso y el tratamiento de la sepsis pueden ser difícil. El programa global, "Campaña Sobrevivir a la Sepsis" (SSC), fue desarrollado en 2002 por organizaciones profesionales para reducir las tasas de mortalidad usando directrices de gestión basadas en la evidencia y estrategias de mejora del rendimiento. Los Las pautas de SSC 2013 están patrocinadas por 30 organizaciones profesionales internacionales.

Elementos clave de las directrices de la CSS de 2013 incluir:

- Reanimación temprana durante las primeras 6 horas de reconocimiento de sepsis.

- Hemocultivos antes del tratamiento con antibióticos. 
- Administración de antibióticos de amplio espectro dentro de 1 hora del reconocimiento de séptico shock o sepsis severa sin choque séptico.

- Pronta ejecución de estudios de imágenes para confirmar fuente potencial de infección.

Reanimación inicial con fluido con cristaloide y considerar la administración de albúmina a mantener la presión arterial media Evitar los esteroides intravenosos si es fluido la reanimación y los vasopresores son capaces de restaurar la estabilidad hemodinámica ${ }^{9,14}$.

Dado que la mayoría de las infecciones necrosantes son polimicrobianos, los antibióticos de amplio espectro temprano son muy recomendables para cubrir grampositivo, gramnegativo y anaeróbico organismos. Escherichia coli es la más patógeno comúnmente aislado involucrado en FG, seguido de Bacteroides, Staphylococcus, Proteus, Streptococcus, Pseudomonas y Enterococcus species. Doble o triple antibioticoterapia con una combinación de cefalosporina de tercera generación, un aminoglucósido, y metronidazol para gramnegativos los aerobios y otros organismos a menudo se usan ${ }^{9,14}$.

Como los antibióticos no pueden penetrar en tejido necrótico que resulta de la necrosis fascitis, es necesario realizar primero un debridamiento quirúrgico. La cobertura de antibióticos puede ser modificada sobre la base de los resultados de intraoperatorio.

\section{MÉTODOS}

El presente trabajo se realizó mediante la revisión sistemática de artículos científicos, revisiones clínicas e informes de casos; tanto en español como en inglés, relacionados con el tema de investigación. Las fuentes de búsqueda empleadas fueron: Google Scholar, ScienceDirect - Elsevier library, PubMed, Scielo. Los términos usados como palabras claves fueron: Gangrena de Fournier, factores de riesgo y factores predisponentes, los cuales fueron unidos por la preposición OR, con la finalidad de delimitar las referencias encontradas, y por ende tener una búsqueda más precisa y exacta al tema a investigar.

Para esta revisión se seleccionaron los artículos publicados que tuvieron experiencias investigativas y teórico-conceptuales. Se analizaron los resultados y se incorporaron los antecedentes en una base de datos para evidenciar similitudes y diferencias que ayuden en la organización del presente artículo.

Los criterios de inclusión utilizados para agrupar los artículos científicos fueron:
1. El título del artículo debía de contener la palabra clave gangrena de Fournier.

2. Debe de contener dentro del artículo el descriptor clave.

3. Los artículos seleccionados debían tener una antigüedad no mayor a 10 años, desde el 2008 a la actualidad.

Los criterios de exclusión utilizados para agrupar los artículos científicos fueron:

1. Que no se desarrolle nada acerca de la gangrena de Fournier.

2. Que los artículos tengan una antigüedad mayor a 10 años.

\section{RESULTADOS}

De los 86 artículos revisados, se descartaron 33 por no estar directamente relacionados con el tema de estudio, y 32 fueron retirados por ser publicados antes del 2008, quedando solo 21 artículos para esta revisión bibliográfica. De los 21 artículos seleccionados, 15 contaban con resultados de investigación e informe de casos; y 6 fueron considerados para aspectos teórico - conceptuales que se relacionan con el propósito de la investigación: Factores de riesgo en gangrena de Fournier.

En el estudio realizado por Novella LC y colaboradores ${ }^{5}$, tuvo como objetivo realizar una revisión de casuísticas de los últimos 10 años y de la enfermedad y su tratamiento. Como resultado no se encontró en los pacientes una etiología clara (salvo en un paciente que había presentado un absceso perianal 20 años antes), pero 3 pacientes (50\% de los casos) presentaban alguna condición predisponente como HTA, cáncer y tabaquismo.

En el estudio retrospectivo de Medina Polo $J \mathrm{y}$ colaboradores $^{14}$, Cakmak A colaboradores ${ }^{20}$, Barreda JT y colaboradores ${ }^{3}$, se tuvo como objetivo evaluar los factores de riesgo y aquellas variables pronósticas que influyen en la supervivencia de los pacientes con gangrena de Fournier. Se revisaron casos donde se determinó que la edad del paciente, la presencia de factores de riesgo, especialmente cáncer, un origen urológico de la infección y la extensión de la enfermedad tienen influencia en el pronóstico de la gangrena de Fournier.

En la investigación tanto de Luján Marco $S$ y colaboradores $^{12}$ y Czymek $\mathrm{R}$ y colaboradores ${ }^{4}$, Martinschek A y colaboradores ${ }^{10}$, Azolas $\mathrm{M}^{2}$ y Altarac $S$ y colaboradores'; $y$ en la revisión del caso descrito por Garcés $C^{21}$, que tenían como objetivo informar sobre 
esta patología y describir los posibles factores de riesgo asociados, se obtuvo resultados similares, donde confirman claramente que la mayoría comorbilidades importantes son condiciones médicas preexistentes o predisposiciones, como diabetes mellitus, crónica alcoholismo, inmovilización prolongada, sobrepeso u obesidad e inmunosupresión. Otras comorbilidades tales como enfermedades cardiopulmonares (hipertensión arterial, enfermedad cardíaca coronaria, insuficiencia cardíaca y EPOC), y consumo de algunos fármacos (AINES) son, sin embargo, no poco comunes en el grupo de edad en riesgo y no deben ser considerados como factores etiológicos.

En estudios más recientes, como el de Oguz A y colaboradores $^{16}$, y loannidis 0 8; se obtuvieron también resultados similares a los descritos, donde una serie de enfermedades sistémicas, incluida la DM, vasculopatía, cirrosis crónica del alcoholismo, insuficiencia renal y el uso de esteroides son factores de riesgo conocidos para el desarrollo de FG. En el primer estudio de casos, la DM fue el factor de riesgo más común, afectando al $40.5 \%$ de los sobrevivientes y $66.7 \%$ de los no sobrevivientes. Aunque algunos estudios no han informado ninguna asociación entre el estado de DM y resultados clínicos en FG, observamos aumento de la mortalidad entre pacientes con DM.

Después de la revisión podemos darnos cuenta que existen múltiples estudios sobre gangrena de Fournier; sin embargo, son pocos los que se evocan exclusivamente al estudio de los factores de riesgo en sí, es por ello que en el presente artículo se busca ahondar un poco más al respecto de modo que se pueda tomar en cuenta estos factores para diagnosticar en los primeros estadios, resaltando en los factores más frecuentes e importantes -por su mortalidad- que podrían orientar nuestro diagnóstico En la Tabla $N^{\circ} 1$, se puede evidenciar de manera precisa que puntos tomas los diferentes autores.

\section{DISCUSIÓN}

No se ha hablado e investigado mucho sobre la gangrena de Fournier, ello debido a que no es una patología frecuente; sin embargo, debe darse el enfoque y la investigación adecuada, ya que si no se trata de manera adecuada y en los primeros estadios es mortal para los pacientes.

A través de la revisión sistemática se tiene en claro que existen diversos factores de riesgo que pueden intervenir en esta patología; empero no se han determinado con exactitud y hay pocos estudios dirigidos a este tema de investigación, pero hay artículos donde coinciden con varios factores de riesgo; sin embargo, difieren o algunos no los consideran en la misma magnitud como posibles factores de morbilidad.

Es por ello que sería importante realizar un estudio exhaustivo sobre los factores de riesgo asociados a la gangrena de Fournier, e incluso también se podría revisar los factores de riesgo postquirúrgicos que también han sido poco descritos.

Por otra parte, en la presente revisión sistemática no se encontró algún artículo publicado en nuestro país respecto al tema; por lo que sería relevante hacer uno y así también corroborar que los factores de riesgo descritos por otros autores también son o no los mismos en nuestra población.

\section{CONCLUSIÓN}

Después de realizada la revisión se evidencia la poca cantidad de estudios dirigidos a hablar específicamente de los factores de riesgo asociados a la gangrena de Fournier, la cual es una patología de gran letalidad y puede limitar el desempeño y afectar la calidad de vida del paciente; por lo que es necesario hacer una mayor investigación sobre este, de modo que se puedan evitar las consecuencias a largo plazo.

La presencia de factores de riesgo contribuye a que la enfermedad se presente con mayor frecuencia, y que si no son tomadas en cuenta pueden pasar desapercibidos, demorando y complicando el diagnóstico y pronóstico de quien la padezca.

La gangrena de Fournier tiene una alta tasa de mortalidad pese a un tratamiento agresivo con tratamiento antibiótico intravenoso y un amplio abordaje quirúrgico resecando la zona afecta. La población de edad más avanzada y aquella que sufre alguna enfermedad debilitante, cualidades que suelen ir asociadas, tienen un peor pronóstico. Además cada vez es más frecuente tratar pacientes de mayor edad. Las tasas de mortalidad más altas son especialmente destacables en los pacientes que padecen algún factor de riesgo. Se trata de una enfermedad que requiere un diagnostico precoz ya que una mayor extensión de la enfermedad también se asocia a un peor pronóstico. 
Tabla 1. Importancia de los trabajos revisados, según autor y año.

\begin{tabular}{|c|c|c|c|c|c|c|}
\hline 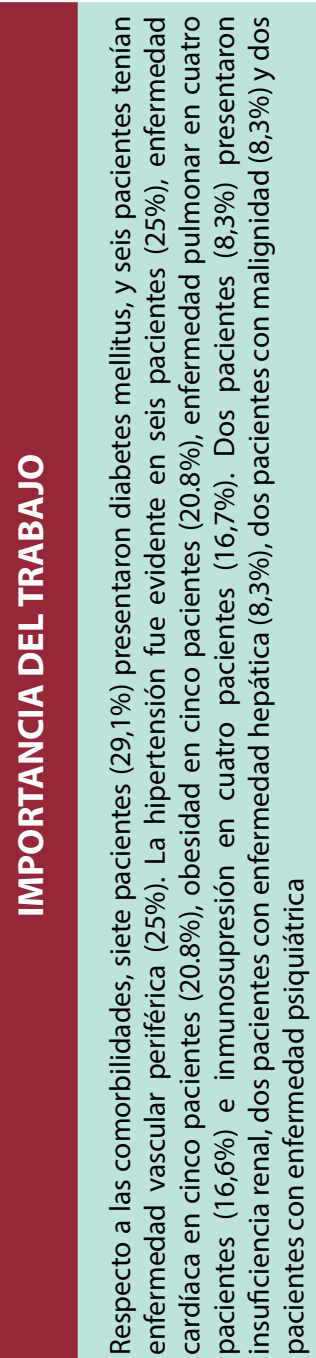 & 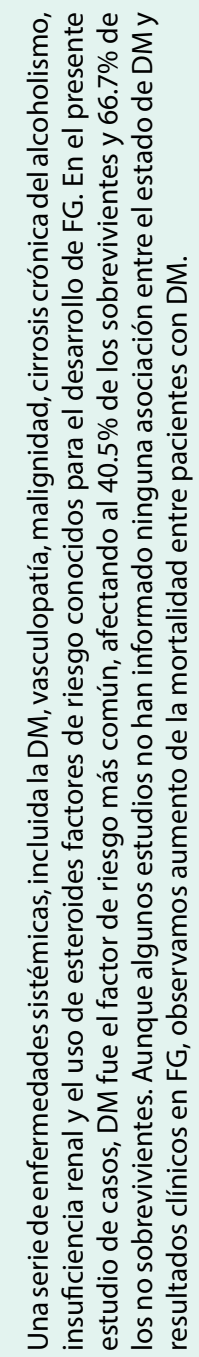 & 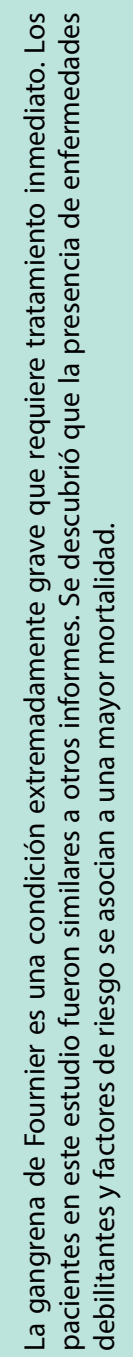 & 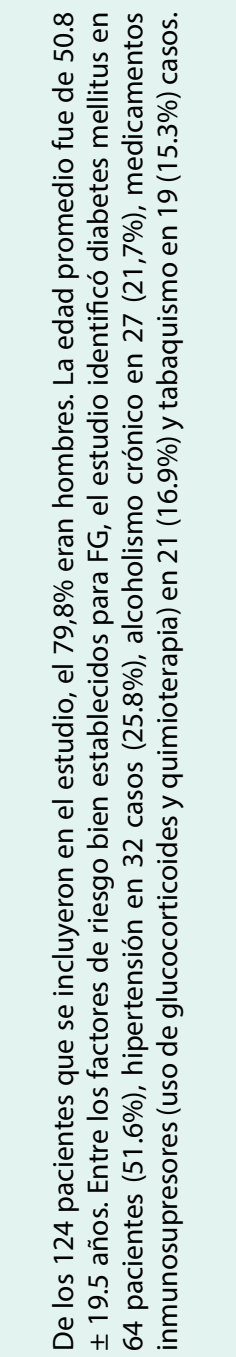 & 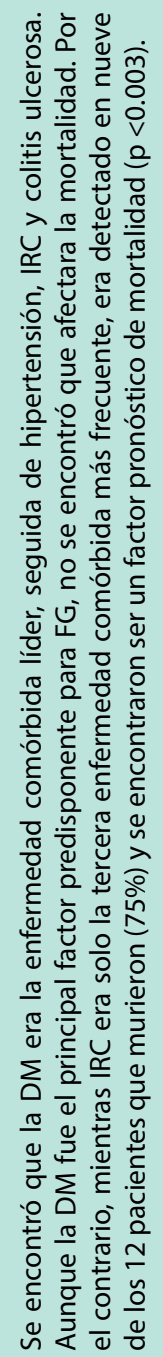 & 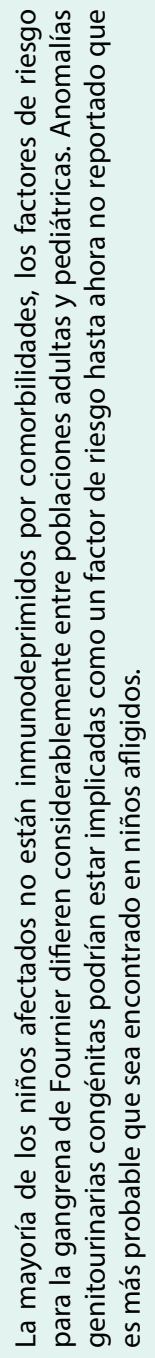 & 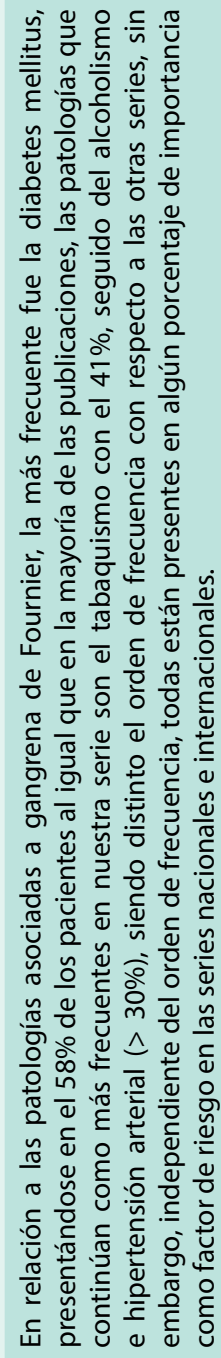 \\
\hline $\begin{array}{l}\frac{0}{3} \\
\frac{0}{5} \\
\frac{5}{5}\end{array}$ & $\frac{\stackrel{0}{J}}{\frac{0}{5}}$ & 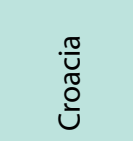 & 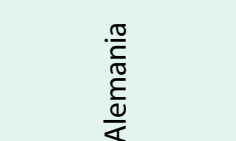 & $\begin{array}{l}\frac{0.0}{5} \\
\frac{5}{5} \\
\frac{5}{5}\end{array}$ & 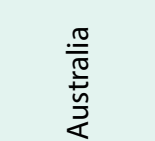 & $\frac{\varrho}{\bar{\tau}}$ \\
\hline 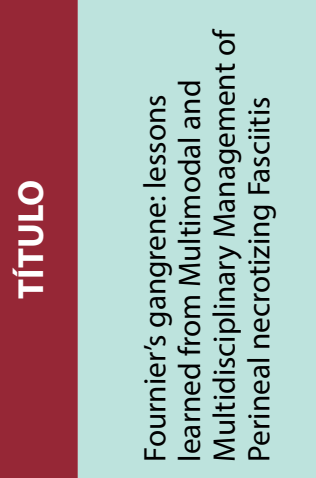 & 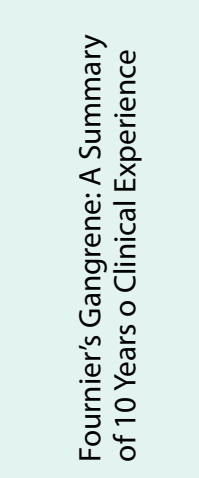 & 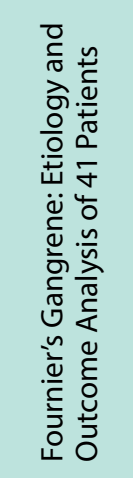 & 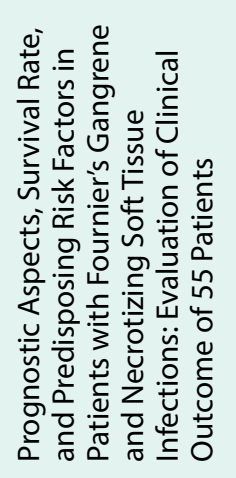 & 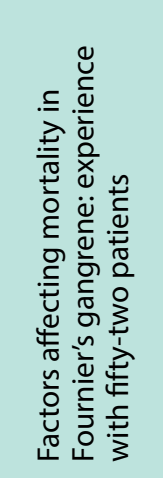 & 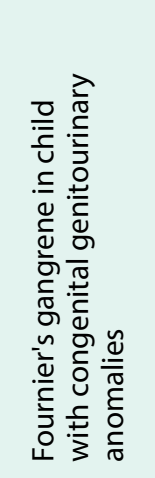 & 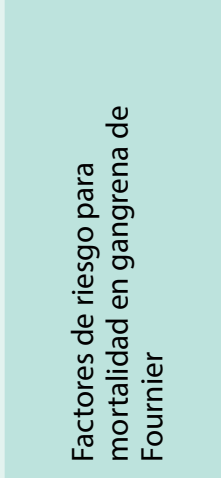 \\
\hline 定 & $\stackrel{n}{\stackrel{n}{N}}$ & $\stackrel{\sim}{i}$ & $\stackrel{\sim}{\stackrel{N}{N}}$ & $\stackrel{\sim}{i}$ & $\stackrel{\sim}{\stackrel{\sim}{*}}$ & $\bar{i}$ \\
\hline 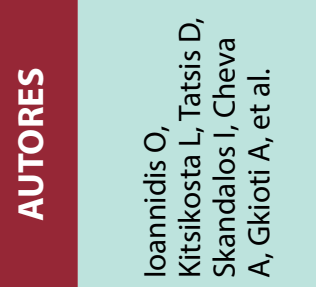 & 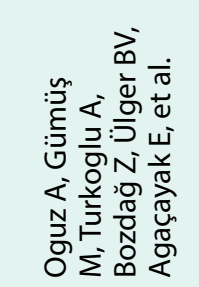 & 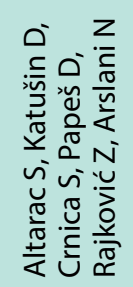 & 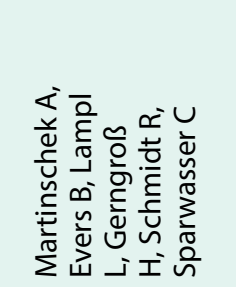 & 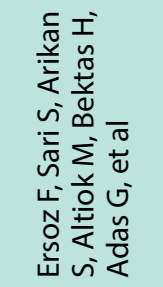 & 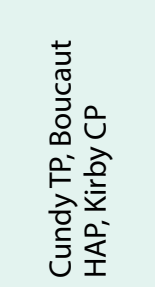 & 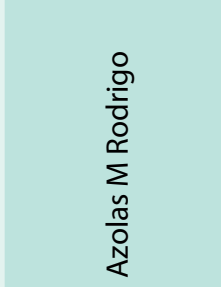 \\
\hline
\end{tabular}




\begin{tabular}{|c|c|c|c|c|c|c|c|}
\hline 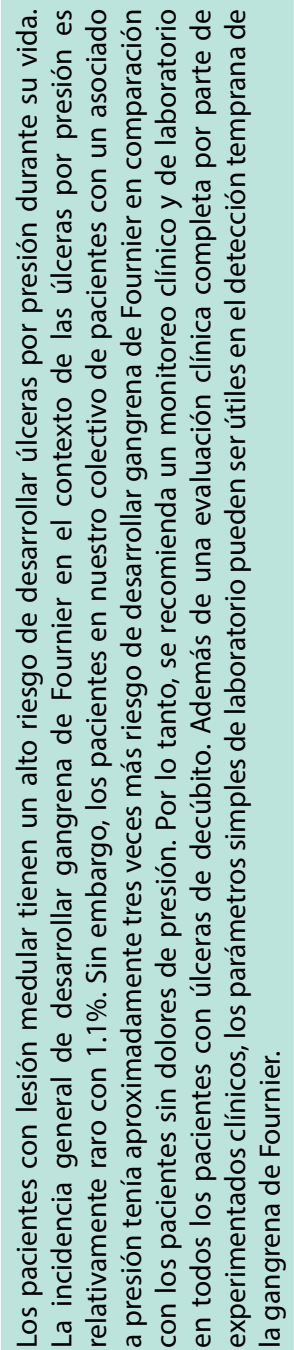 & 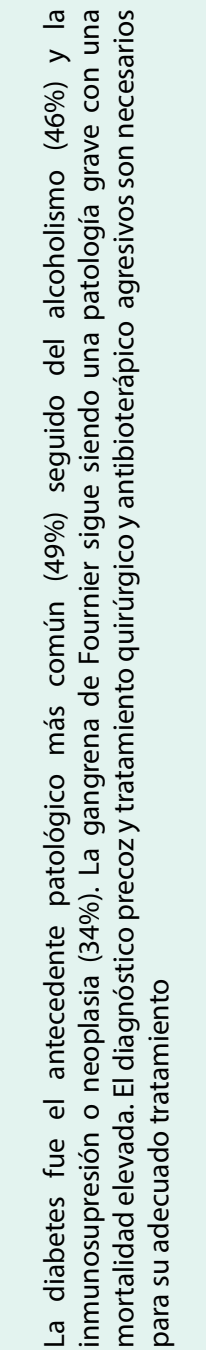 & 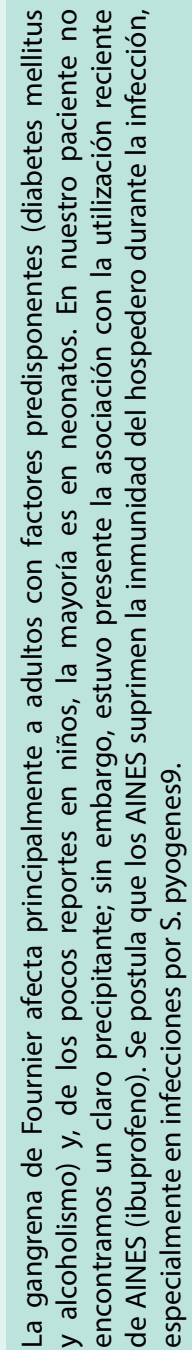 & 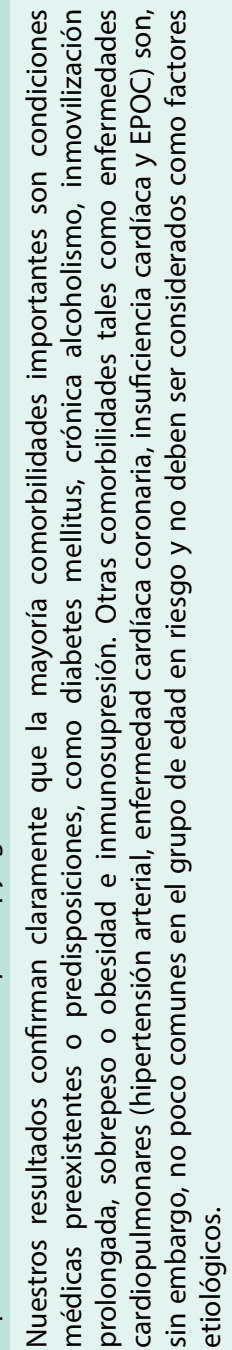 & 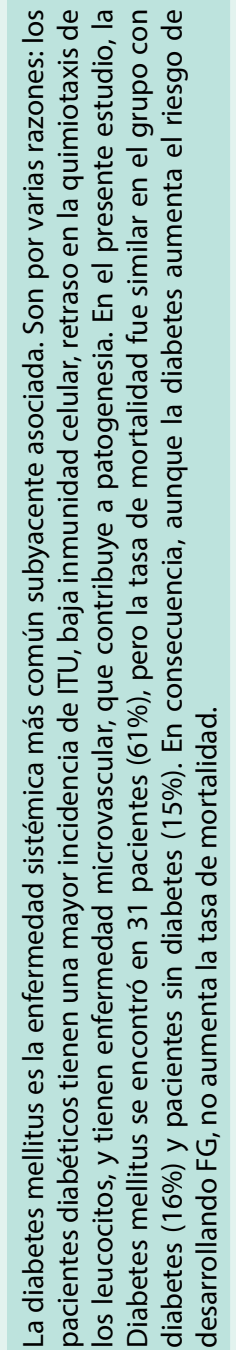 & 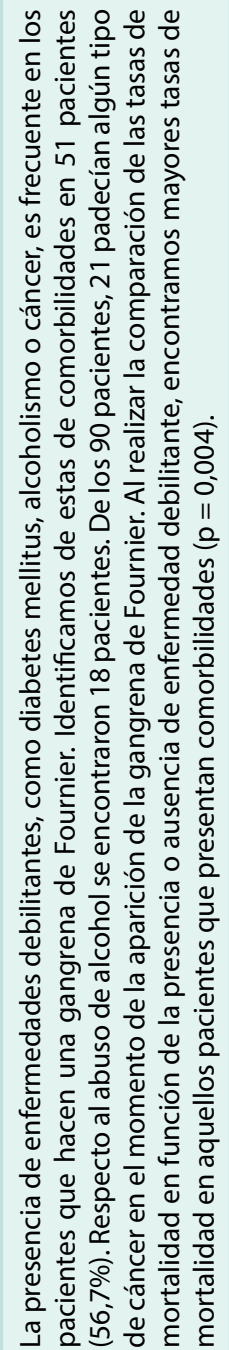 & 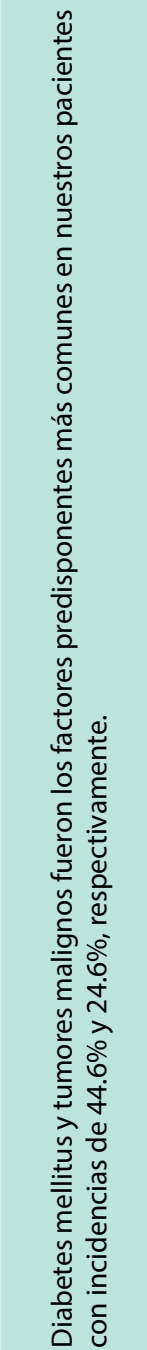 & 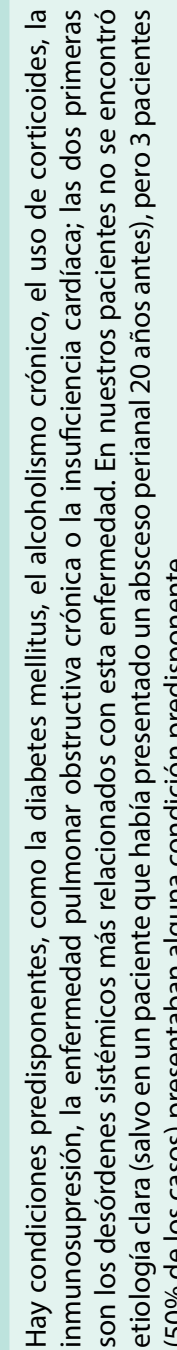 \\
\hline 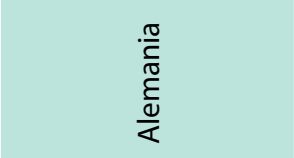 & 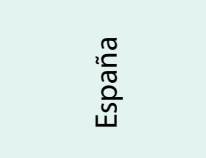 & $\begin{array}{l}\frac{\pi}{0} \\
\frac{0}{\varepsilon} \\
\frac{0}{0}\end{array}$ & 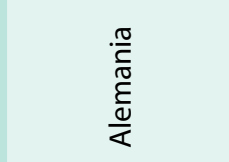 & 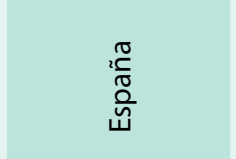 & 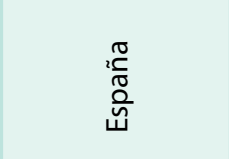 & $\begin{array}{l}\frac{0}{5} \\
\frac{0}{5} \\
\frac{5}{5}\end{array}$ & 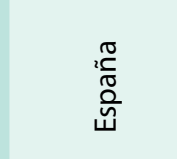 \\
\hline 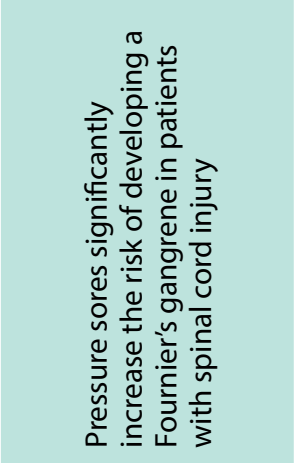 & 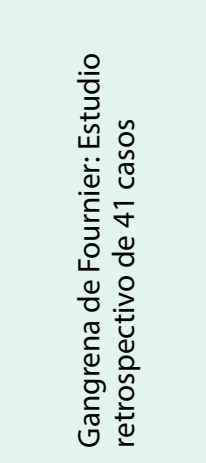 & 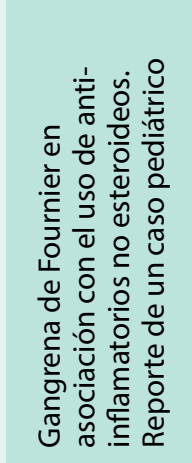 & 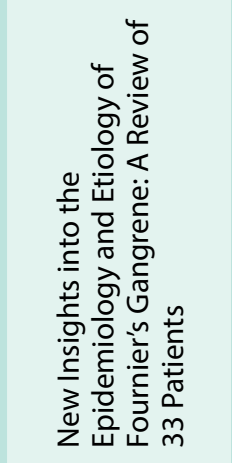 & 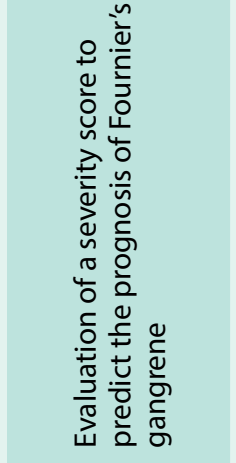 & 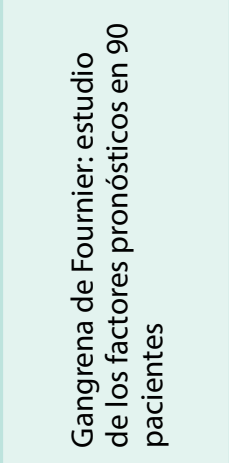 & 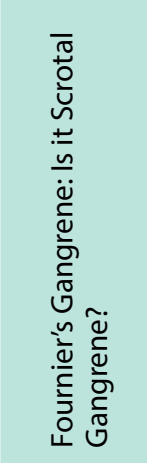 & 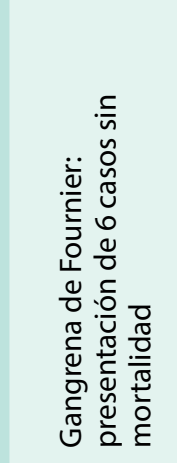 \\
\hline $\bar{i}$ & $\frac{\circ}{i}$ & $\stackrel{\circ}{\circ}$ & ஓे & ᄋి & $\stackrel{\infty}{\stackrel{\sim}{~}}$ & $\stackrel{\infty}{\stackrel{\nu}{\circ}}$ & $\stackrel{\infty}{\circ}$ \\
\hline 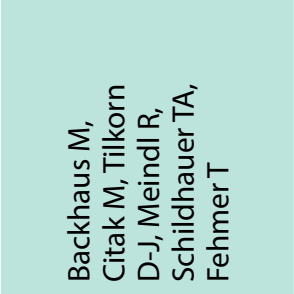 & 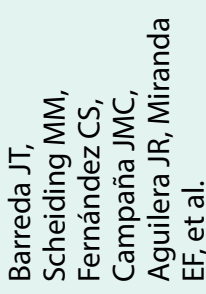 & 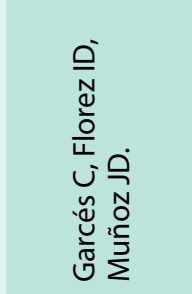 & 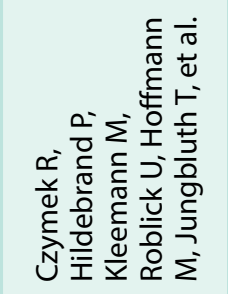 & 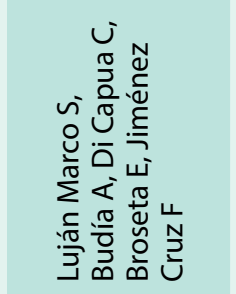 & 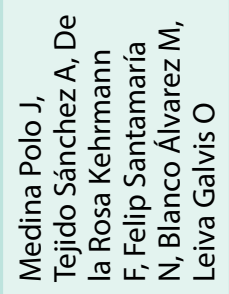 & 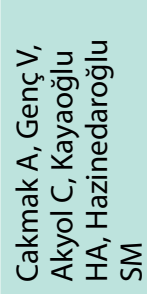 & 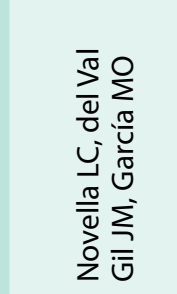 \\
\hline
\end{tabular}


Contribuciones de autoría: El autor participó en la generación, recolección de información, redacción y aprobación final del artículo original.

Financiamiento: Autofinanciado.

Conflicto de interés: El autor declara no tener conflicto de interés en la publicación de este artículo.

Recibido: 26 de julio del 2018

Aprobado: 25 de setiembre del 2018

Correspondencia: Jose C. Regalado Altamirano

Dirección: Av. Pacífico N¹80 dpto 1104, San Miguel-Parques de la Huaca

Celular: 923136252

Correo:jose_17u@hotmail.com

\section{REFERENCIAS BIBLIOGRÁFICAS}

1. Altarac S, Katušin D, Crnica S, Papeš D, Rajković Z, Arslani N. Fournier's gangrene: etiology and outcome analysis of 41 patients. Urol Int. 2012;88(3):289-93.

2. Azolas R. Factores de riesgo para mortalidad en gangrena de Fournier Rev Chil Cir. 2011;63(3):270-275.

3. Barreda JT, Scheiding MM, Fernández CS, Campaña JMC, Aguilera JR, Miranda EF, et al. Gangrena de Fournier: Estudio retrospectivo de 41 casos. Cirugia Espanola. 2010;87(4):218-223.

4. Czymek R, Hildebrand P, Kleemann M, Roblick U, Hoffmann M Jungbluth $\mathrm{T}$, et al. New insights into the epidemiology and etiology of Fournier's gangrene: a review of 33 patients. Infection. agosto de 2009;37(4):306-12.

5. Novella LC, del Val Gil JM, García MO. Gangrena de Fournier presentación de 6 casos sin mortalidad. Cir Esp. 2008;84(1):28-31.

6. Montoya Chinchilla R, Izquierdo Morejon E, Pietricicâ BN, Pellicer Franco E, Aguayo Albasini JL, Miñana López B. Gangrena de Fournier: Análisis descriptivo de 20 casos y revisión de la bibliografía científica. Actas Urol Esp. 2009;33(8):873-880.

7. Kincius M, Telksnys T, Trumbeckas D, Jievaltas M, Milonas D. Evaluation of LRINEC Scale Feasibility for Predicting Outcomes of Fournier Gangrene. Surg Infect. agosto de 2016;17(4):448-53.

8. Ioannidis O, Kitsikosta L, Tatsis D, Skandalos I, Cheva A, Gkioti A et al. Fournier's Gangrene: Lessons Learned from Multimodal and Multidisciplinary Management of Perineal Necrotizing Fasciitis. Front Surg [Internet]. 10 de julio de 2017 [citado 9 de mayo de 2018];4. Disponible en: https://www.ncbi.nlm.nih.gov/pmc/articles/PMC5502266/

9. Kaufmann JA, Ramponi D. Recognition of risk factors and prognostic indicators in Fournier's gangrene. Crit Care Nurs Q. junio de 2015;38(2):143-53.

10. Martinschek A, Evers B, Lampl L, Gerngroß H, Schmidt R, Sparwasser C. Prognostic aspects, survival rate, and predisposing risk factors in patients with Fournier's gangrene and necrotizing soft tissue infections: evaluation of clinical outcome of 55 patients. Urol Int. 2012;89(2):173-9.
11. Cundy TP, Boucaut HAP, Kirby CP. Fournier's gangrene in a child with congenital genitourinary anomalies. J Pediatr Surg. abril de 2012;47(4):808-11.

12. Luján Marco S, Budía A, Di Capua C, Broseta E, Jiménez Cruz F. Evaluation of a severity score to predict the prognosis of Fournier's gangrene. BJU Int. agosto de 2010;106(3):373-6.

13. Ersoz F, Sari S, Arikan S, Altiok M, Bektas H, Adas G, et al. Factors affecting mortality in Fournier's gangrene: experience with fifty-two patients. Singapore Med J. agosto de 2012;53(8):537-40.

14. Medina Polo J, Tejido Sánchez A, De la Rosa Kehrmann F, Felip Santamaría N, Blanco Álvarez M, Leiva Galvis O. Gangrena de Fournier: estudio de los factores pronósticos en 90 pacientes. Actas Urol Esp. 2008;32(10):1024-1030.

15. Múñoz C, Herrera KFP, Cesín L. Gangrena de Fournier: reporte de un caso. Rev Hispanoam Cienc Salud RHCS. 2017;3(3):103-106

16. Oguz A, Gümüş $M$, Turkoglu A, Bozdağ Z, Ülger BV, Agaçayak $E$, et al. Fournier's Gangrene: A Summary of 10 Years of Clinical Experience. Int Surg. mayo de 2015;100(5):934-41.

17. Roghmann F, von Bodman C, Löppenberg B, Hinkel A, Palisaar J, Noldus $J$. Is there a need for the Fournier's gangrene severity index? Comparison of scoring systems for outcome prediction in patients with Fournier's gangrene. BJU Int. noviembre de 2012;110(9):1359-65.

18. Shyam DC, Rapsang AG. Fournier's gangrene. The Surgeon. agosto de 2013;11(4):222-32.

19. PEÑA JLP. PREVALENCIA DE CELULITIS EN EL SERVICIO DE PEDIATRÍA DEL HOSPITAL MARÍA AUXILIADORA DESDE EL AÑO 2009 - AÑO 2014. :35.

20. Cakmak A, Genç V, Akyol C, Kayaoğlu HA, Hazinedaroğlu SM. Fournier's gangrene: is it scrotal gangrene? Adv Ther. octubre de 2008;25(10):1065-74.

21. Garcés C, Florez ID, Muñoz JD. Gangrena de Fournier en asociación con el uso de anti-inflamatorios no esteroideos: Reporte de un caso pediátrico. Rev Chil Infectol. 2010;27(4):341-344. 